\title{
DA SAN NILO ALL'AFFERMAZIONE DEL MONACHESIMO LATINO IN CALABRIA
}

\section{ANTONIO MACCHIONE}

UDC: $726.71(450.78) " 08 / 10 "$

Original scientific paper

Manuscript received: 04. 11. 2016.

Revised manuscript accepted: 08. 02. 2017.

DOI: 10.1484/J.HAM.5.113725
A. Macchione

Università della Basilicata

Via Nazario Sauro, 85

85100 (Potenza), Italia

ant.macchione75@gmail.com

The story of Calabria, between the ninth and eleventh centuries, was marked by the coexistence of different political and religious souls. Along the Valley of the Crati (up to Cosenza) the Lombard presence came while, further south, the Byzantine people acted with the formation of the Thema of Calabria. In addition, the migration waves of the eighth century led to the 'bizantinizzazione' of church and monastic structures. But, since the second half of the ninth century, the influence of Montecassino archcenoby began to put some full stops, in the slow process of recovery of the South to the Roman obedience and to the liturgical latinity. This gave birth to the integration processes of the two religious components; but with the spread of the Congregation Cavense (1025), the Italo-Greek monastic component, especially that along the border and in the area of Calabria and Lucania of Mercurion, ended with being more and more incorporated into the Latin monastic reorganization. This complex situation, since the second half of the eleventh century with the arrival of the Normans, underwent a profound transformation by recording the natural rotation of new bishops of the Latin Rite with those of Greek rite and the founding of new Benedictine abbeys for the feudal and spiritual control of the territory (1062-1091).

Keywords: Nilo di Rossano, Montecassino, SS. Trinità di Cava, Normanni, Calabria

Tra IX e XI secolo, la Calabria è una terra di confine in cui convivono anime diverse sia dal punto di vista politico che religioso'. Infatti, se lungo la Valle del Crati (fino a Cosenza) è ben attestata la presenza longobarda, sotto questa linea agiscono i bizantini che in seguito alla formazione del Thema di Calabria, nato sulle ceneri dell'antico Ducato di VI secolo, detengono il controllo amministrativo e strategico dei traffici e delle rotte commerciali con l'Oriente e con la Sicilia islamizzata. Da quest'ultima, schiere di monaci di culto italo-greco, si apprestano a guadagnare il continente incalzati dall'avanzata saracena ${ }^{2}$. Del resto il fatto che il Mezzogiorno sia stato un luogo privilegiato per l'incontro tra fedi, culture ed etnie differenti, è un dato storiografico oramai stabilmente acquisito 3 .

Dalla fine dell'VIII secolo le ondate migratorie monastiche avevano determinato la 'bizantinizzazione' delle strutture ecclesiastiche in tutta la regione ma, negli stessi anni, sotto l'influenza dell'archicenobio cassinese, comin- ciava un processo analogo ma inverso per cui l'espansione dei possedimenti cassinesi favoriva il recupero cultuale di ampie regioni del Mezzogiorno continentale, già grecizzate.

E se la presenza dei monaci italo greci era attestata dalle Saline, al Kellerana e fino alle macro-aree del Mercurio e del Latiniano (tra Calabria settentrionale e Lucania meridionale), coprendo omogeneamente quasi tutto il territorio regionale, la presenza cassinese cominciava ad insinuarsi nella Valle del Crati, per guadagnare la costa sino a Cetraro e spingersi poi verso Tropea ${ }^{4}$; mentre l'espansione della Congregazione Cavense (1025) ampliava quel primitivo orizzonte sostituendosi gradatamente, lungo il confine calabro-lucano, alle enclaves monastiche italo greche inglobandone le unità produttive (mulini, gualchiere, trappeti ecc.), oltre che i beni rurali. Questa complessa situazione, a partire dalla seconda metà dell'XI secolo con l'arrivo dei normanni, subì una profonda trasformazione registrando il naturale avvicendamento di nuovi vescovi di rito latino a quelli di

\footnotetext{
${ }^{1}$ La Calabria, rientrava nel novero delle regioni appartenute al cosiddetto Impero romano d'Oriente, un'entità statale «che con un'espressione non felice, ma oramai generalmente accettata, si è soliti chiamare Impero bizantino». Dalla sua civiltà i territori dell'Italia meridionale ricevettero «un'impronta - più o meno profonda, visibile e duratura secondo i luoghi e le loro vicissitudini storiche - nella lingua, nella cultura letteraria ed artistica, nella vita sociale, nell'organizzazione del culto e nelle tradizioni religiose e spirituali» (E. FOLLIERI, I santi dell'Italia greca, in Histoire et culture dans l'Italie byzantine, curr. A. Jacob et alii, Rome, 2006 (Collection de l'Ėcole française de Rome, 363), p. 95. Per un inquadramento storico generale, oltre ai classici lavori di J. GAY, L'Italie méridionale et l'empire byzantine depuis l'avènement de Basile I Ier jusqu'à la prise de Bari par les Normands (867-1071), Paris, 1904 (Bibliothèque des Ėcoles françaises d'Athènes et de Rome, 9o); si vd. il contributo di A. GUILLOU, Aspetti della civiltà bizantina in Italia, Bari, 1976; V. VON FALKENHAUSEN, La dominazione bizantina nell'Italia meridionale dal IX all'XI secolo, Bari, 1978; F. BURGARELLA, Le terre bizantine, in Storia del Mezzogiorno, dir. G. Galasso e R. Romeo, II.2, Napoli, 1989, p. 414-517; più di recente ID., La Calabria bizantina (VI-XI secolo), in San Nilo di Rossano e l'abbazia greca di Grottaferrata, cur. Id., Roma, 2009, p. 19-38.

${ }^{2}$ Vita di Sant'Elia il Giovane, cur. G. Rossi Taibbi, Palermo, 1962, p. 36-37, c. 25.

3 Una lucida sintesi sul tema in F. PANARELLI, Aspetti della molteplicità etnica nel monachesimo del Mezzogiorno normanno, in Quellen und Forschungen aus italianischen Archiven und Bibliotheken, 85 (2005), p. 74-103.

${ }^{4}$ Di recente Annick Peters Custot ha sostenuto che prima dell'epoca normanna in Calabria non vi era traccia di monasteri latini. Questa affermazione, alla luce dell'edizione del Registrum Petri Diaconi curata da J.M. Martin et alii sembra essere messa in discussione da alcuni documenti che attestano l'esistenza di una dipendenza cassinese nella città di Cosenza. Come meglio si vedrà in seguito è opportuno rivedere questa posizione anche se è da escludere la presenza diffusa del rito latino e sicuramente è accettabile quanto sostiene la stessa Peters Custot nell'affermare che «a fortiori le monachism latin, dans les pays hellénisés, devait-il se limiter à quelques fondations urbaines» [A. PETERS CUSTOT, Les grecs de l'Italie méridionale post-byzantine une acculturation en douceur, Rome, 2009, (Collection de l'Ėcole française de Rome, 420), p. 268].
} 
rito greco e la fondazione di nuove abbazie benedettine per il controllo feudale e spirituale del territorio (1062-1091) ${ }^{5}$.

\section{QUADRI MONASTICI ITALO GRECI IN CALABRIA}

I numerosi, e spesso minuscoli, monasteri italo greci componevano un mosaico insediativo le cui tessere erano sparse in tutte le province della regione. Accanto a cellule monastiche di piccole dimensioni, godevano di maggior prestigio e notorietà i complessi monastici delle Saline legati alla figura di s. Elia il Giovane ${ }^{6}$, tra i quali il monastero del Kellerana fondato sotto la tuitio spirituale dell'abate Nicodemo; i monasteri di Bruzzano, Sinopoli, Melicuccà e Pedavoli; e quelli del Mercourion e del Latiniano in cui fiorirono le esperienze ascetiche di Nilo di Rossano, Fantino il Giovane, Bartolomeo Iuniore, Saba e Macario (per citare i più importanti). Solo la parte centrale della regione, rimase a lungo priva di riferimenti certi ad accezione dell'area del Carrà (un fitto recesso boschivo tra Nicastro e Catanzaro) in cui furono concentrati tutta una serie di monasteri (anche imperiali) come risulta dal resoconto di Atanasio Calceopulo7. L'organizzazione monastica non seguiva un'unica regola ma ogni monastero aveva un proprio typicon, spesso mutuato dalla diataxeis di Basilio Magno in cui erano evidenti i riferimenti alla Regula Magistri di tradizione benedettina ${ }^{8}$.

I Tipikà prescrivevano uno stile di vita monastico in cui i tempi della preghiera erano alternati al riposo e al lavoro negli scriptoria e nei campi, di cui i monasteri possede- vano ampie porzioni. Del resto, lo dimostra chiaramente la documentazione superstite, alle fondazioni monastiche italo-greche oltre che il controllo spirituale del territorio, era assegnato anche quello economico e sociale nonché un importante ruolo di intermediazione con le autorità politiche 9 .

Infatti, i Bioi dei santi monaci italo greci tramandano fondamentali notizie sulla devozione delle popolazioni locali che affidavano agli uomini di Dio le loro vicissitudini di natura pratica, demandando loro il compito - spesso non facile - di intercedere con le autorità politiche. D'altro canto le agiografie ambientavano le narrazioni in paesaggi aspri e difficili da sottomettere, nei quali monaci, asceti e contadini, lavoravano quotidianamente fianco a fianco per addomesticare il territorio, strappando al bosco e alla foresta, plaghe di terreno fertile per l'agricoltura ${ }^{10}$.

Lasperità del paesaggio fisico rifletteva tanto i tentativi di integrazione, che il forte contrasto etnico tra popolazioni, stigmatizzate nelle vivaci tensioni e nei contrastanti rapporti tra monaci italo greci e nobiltà normanna all'indomani della conquista. Ad esempio Luca di Isola Capo Rizzuto ammonisce il nobile normanno Revetos ${ }^{11}$ intimandogli di non ridurre i sacerdoti in schiavitù. Quest'ultimo, colpito da una grave malattia, abbandona i cattivi propositi e viene miracolosamente guarito dopo un pellegrinaggio alla tomba del santo monaco, ma muore qualche tempo dopo quando, dimentico del beneficio ricevuto, ritorna a molestare i sacerdoti di rito greco ${ }^{12}$.

\footnotetext{
${ }_{5}$ Sulla politica di rekatholisierung si vd. L.R. MÉNAGER, La «byzantinisation» religeuse de l'Italie méridionale (IX'-XIIe siècles) et la politique monastique des Nomands d'Italie, in Revue d'histoire ecclésiastique, LIV (1959), p. 5-40; ID., Les fondations monastiques de Robert Guiscard, duc de Pouille et de Calabre, in Quellen und Forschungen aus italienischen Archiven und Bibliotheken, 39(1959), p. 1-116; E. PONTIERI, Tra i normanni nell'Italia meridionale, Napoli, $1964^{2}$; C.D. FONSECA, La prima generazione normanna e le istituzioni monastiche dell'Italia meridionale, in Roberto il Guiscardo e il suo tempo, Relazioni e comunicazioni nelle Prime giornate normanno-sveve (Bari, maggio 1973), Roma, 1975, p. 135-146; H. HOUBEN, Roberto il Guiscardo e il monachesimo, in Benedictina, 32 (1985), p. 495-520, poi in ID., Medioevo monastico meridionale, Napoli, 1987, p. 109-127.

${ }^{6}$ Per l'eparchia delle Saline si vd. A. GUILLOU, La tourma des Salines dans le thème de Calabre (XIe siècle), in Mélanges de l'École française de Rome. Moyen

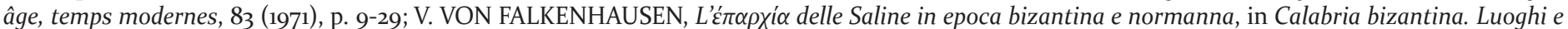
circoscrizioni amministrative, Reggio Calabria, 2009, p. 89-105; E. MORINI, La santità nella Valle delle Saline, Ibidem, p. 291-323.

${ }^{7}$ M.H. LAURENT, A. GUILLOU, Le 'Liber visitationis' d'Athanase Chalkéopoulos (1457-1458). Contribution à l'histoire du monachisme grec en Italie méridionale, Città del Vaticano, 1960. Sul Liber visitationis si vd. A. VARVARO, Capitoli per la storia linguistica dell'Italia meridionale e della Sicilia, IV. Il Liber visitationis di Atanasio Calceopulo (1457-1458), in Medioevo romanzo, XI (1986), p. 55-110. La vicenda dei monasteri del Carrà è stata ricostruita da F.A. PARISI, I monasteri basiliani dell'istmo di Catanzaro, in Archivio storico per le province napoletane, 75 (1956), p. 165-202.

${ }^{8}$ I typikà sono le regole esemplate dagli abati che si richiamavano alla diataxeis di Basilio Magno e si ispiravano alle codificazioni imperiali. Ogni monastero aveva il suo typikon e, spesso, alcuni ne adottavano più d'uno, o piuttosto un insieme di testi giuridici, disciplinari, liturgici, per regolare l'ascesi e la disciplina monastica. Essi contengono anche molte indicazioni per organizzare e gestire la quotidianità monastica. A tal proposito si vd. H. HOMONT, Le Typicon de S. Nicolas de Casole prèes d'Otrante, in Revue des études grecques, III (189o), p. 381-391; T. MINISCI, I Typikà liturgici dell'Italia bizantina, in Bollettino della Badia Greca di Grottaferrata, VII (1953), p. 97-104; A. PERTUSI, Aspetti organizzativi e culturali dell'ambiente monacale greco dell'Italia meridionale, in L'Eremitismo in Occidente nei secoli XI e XII, Atti della seconda settimana internazionale di studi (Mendola, 30 agosto-6 settembre 1962), Milano, 1965, p. 382-426; M. ARRANZ, Le typicon du monastère du Saint Sauveur à Messine. Codex Messinensis gr. 115, A.D. 1131, Roma, 1969; F. BURGARELLA, Aspetti del monachesimo greco nella Calabria bizantina, in Per una idea di Calabria, Atti del convegno (Cosenza, 27-28 novembre 1981), Cosenza, 1982, p. 55-71. ${ }^{9} \mathrm{Si}$ vd. il caso di Sant'Elia il Giovane che, interpellato sull'esito della battaglia di Taormina (889-89o) da Michele stratega di Calabria, consigliò all'uomo d'armi di far purificare il popolo. Michele segui i consigli del santo monaco, sconfisse i nemici e «dopo questi avvenimenti (...), diceva che Elia era veramente servo di Dio. Se infatti non fosse stato amato da Dio, non avrebbe potuto preannunziare tali fatti, né dare tali ammaestramenti» [Vita di S. Elia il Giovane, op. cit. (n. 2), p. 63, c. 43]. Si vd. anche l'intercessione di Bartolomeo Iuniore: questi commosso dalle lacrime dei famigliari di Adenolfo, duca di Gaeta, intraprese un lungo viaggio da Grottaferrata sino a Salerno per impetrarne la liberazione al principe Guaimario V (Vita di S. Bartolomeo Iuniore, cur. G. Giovannelli, Grottaferrata, 1962, p. 34-35, cc. 11-12). Sul rapporto tra potere e santità è ricco di spunti il contributo di A. CILENTO, Potere e monachesimo. Ceti dirigenti e mondo monastico nella Calabria bizantina (secoli IX-XI), Firenze, 2000, p. 89-130.

${ }^{10}$ Nella vita di Sant'Elia lo Speleota si racconta di monaci che tagliavano alberi sulla cima del monte sopra Seminara, rotolando i tronchi a valle insieme a grossi sassi dei quali non si conosce l'utilizzazione finale; oppure si può fare riferimento al Mercourion, vera e propria città monastica realizzata a spese della foresta. È noto per quest'ultima il duro lavoro dei confratelli di Nilo di Rossano, ottimi pescatori, intenti ad intrecciare ceste o a recidere e bruciare alberi per dissodare il terreno e renderlo idoneo alla produzione di frumento necessario al sostentamento degli asceti (Vita di San Nilo di Rossano, fondatore e patrono di Grottaferrata, cur. G. Giovannelli, Grottaferrata, 1966, p. 48, c. 31; 55, c. 38; 59-6o, c. 43).

${ }^{n}$ Forse da identificare col signore normanno Roberto Ravatos, menzionato in un coevo documento di Mileto, v. L.-R. MENAGER, L'abbaye bénédectine de la Trinità de Mileto en Calabre à l'époque normande, in Bullettino dell'archivio paleografico italiano, n.s., IV-V (1958-1959), p. 47-49, n. 16.

${ }^{12}$ Vita di San Luca, vescovo di Isola Capo Rizzuto, cur. G. Schirò, Palermo, 1954, p. 120-122.
} 
L'esempio dimostra che gli agiografi, non discostandosi dai coevi modelli bizantini, presentano i santi monaci sotto una duplice veste: essi sono sia campioni della santità e strumenti di coesione sociale; che potenti taumaturghi, camminatori infaticabili, rigorosi asceti. Appartiene all'apparato narrativo anche lo stringente legame tra monaco e territorio, e tra monaco e comunità rurale definendo le relazioni con gli uomini del potere. Questo fa si che essi si trasformino in un strumento di autorappresentazione delle dinamiche di convivenza nelle campagne calabresi ${ }^{13}$. Un noto episodio, in tal senso, è tratto dalla vita di San Nilo di Rossano. Questi, in contrasto con Euprassio krités di Calabria, per il possesso di alcuni beni fondiari, è supplicato dallo stesso funzionario affinché lo guarisca da una fastidiosa malattia ai genitali. Nilo, dopo lunga insistenza, esaudisce il volere del notabile cui è restituita l'integrità fisica. L'episodio sembra testimoniare che i poteri taumaturgici dei monaci si trasformano in elemento di perequazione sociale. Per cui è nell'orizzonte di una profonda spiritualità, infarcita di miracoli e costellata di prodigi, che si ricompone l'identità delle comunità rurali della Calabria medievale nelle loro componenti sociali'it

Strettamente legato a ciò è il tema del profetismo che, fungendo spesso da cornice narrativa per il racconto, non serve soltanto a pronosticare l'esito di battaglie né ad annunciare invasioni apocalittiche, ma rivela interventi monastici organizzativi e diplomatici a fianco delle autorità militari del tempo per pianificare strategie difensive in territori soggetti a frequenti incursioni saracene. Molto interessante in tal senso la profezia di San Fantino, narrata nella vita di san Nilo, circa la devastazione del Mercurio (950-952) ad opera dei saraceni, la sua 'fuga' nel salernitano e la conseguente diaspora monastica verso i castelli «che loro capitavano» ${ }^{15}$.

\section{L'EPARCHIA DEL MERCURIO}

Un importante centro di spiritualità italo-greca è senza dubbio l'eparchia ${ }^{16}$ mercuriense definita da Oreste patriarca di Gerusalemme, nelle vite dei santi Cristoforo, Saba e
Macario di Collesano, una estesa provincia monastica, incuneata tra i confini di Calabria e Longobardia, al limite dell'impero bizantino e del principato di Salerno, «tutta ammantata di folte e aspre boscaglie popolate da una moltitudine di anacoreti di ambo i sessi $\aleph^{17}$. Analogamente il biografo di S. Leoluca di Corleone, soffermandosi sul passaggio dell'eremita dal monte Mula al Mercurio, paragona l'insediamento ad una 'nuova Tebaide', fucina spirituale in cui temprarono la loro vocazione numerose schiere monastiche, fra le quali spiccano le personalità di Fantino il Giovane e Nilo di Rossano ${ }^{18}$. L'importanza e la centralità dell'insediamento mercuriense crebbe soprattutto dopo la caduta di Taormina in mano agli arabi (902), trasformandosi in luogo di residenza, o passaggio obbligato, per i monaci provenienti dalla Sicilia, come sembrano dimostrare anche i racconti agiografici di san Luca di Demenna e di san Vitale da Castronuovo ${ }^{19}$.

L'habitat mercuriense, tuttavia, non è caratterizzato soltanto da ampie distese boschive e da recessi montuosi inesplorabili in cui esercitare l'ascesi e la solitudine; ma è formato da ampie zone coltivate a cereali, vigneti ed orti con alberi da frutto nelle parti più vicine al mare. Nel Bios di S. Nilo si fa riferimento, addirittura, ad una piccola industria per la produzione di cesti, cestini e panieri in vimini o in canne e paglia la cui tecnica era stata anche trasmessa ad alcuni monaci ${ }^{20}$. E, nello stesso Bios, si fa riferimento alla pratica della pesca, lungo i corsi d'acqua, esercitata dagli abitanti del luogo e dai monaci ${ }^{21}$. Quest'ultimo elemento permette di localizzare il monastero di $S$. Fantino, lungo la valle dell'Argentino, in quanto Bartolomeo Iuniore ritiene che Nilo dalla cella vedesse quotidianamente i monaci pescare ${ }^{22}$.

Lo spazio monastico, a mano a mano, si trasformò in un'ampia comunità facente capo ad un eparca ${ }^{23}$; si costruirono eremitaggi, laure e cenobi plasmando così una nuova entità militare indipendente. Corrispondeva all'eparchia mercuriense anche il territorio di Malvito, un preesistente centro longobardo (forse un gastaldato), sul quale erano installati alcuni monasteri tra cui quello più noto dei Siracusani, frequentato da Saba di Collesano ${ }^{24}$.

\footnotetext{
${ }_{13}^{13}$ Spesso tutto ciò generava attriti tra funzionari e monaci in competizione per questione di prestigio e di rappresentanza (Vita di San Nilo di Rossano, fondatore e patrono di Grottaferrata, cur. G. Giovannelli, Grottaferrata, 1966, p. 51, c. 5; 90-93, c. 47-49; anche infra n. 14).

${ }^{14}$ Vita di San Nilo, op. cit. (n. 10), p. 95, c. 53; 96-99, c. 55-57.

${ }^{15}$ Ibidem, p. 41-42, c. $24 ; 46-47$, c. 29.

${ }^{16}$ Il termine, secondo Filippo Burgarella, aveva assunto nel linguaggio della Calabria greca tra X e XI secolo una connotazione antiquaria designando una circoscrizione amministrativa soggetta alla sovranità imperiale e nel quale «in presenza di un ordinamento tematico oramai compiutamente instaurato, si ha motivo di riconoscere una turma, suddivisione territoriale e militare di una provincia più ampia e di dimensioni regionali quale era appunto il tema» [F. BURGARELLA, L'eparchia di Mercurio: territori e insediamenti, in Rivista di studi bizantini e neoellenici, n.s. 39 (2002), p. 59-92: 6o-61; A. GUILLOU, La Lucanie byzantine. Étude de géographie historique, in Byzantion, 35 (1965), p. 119-149: 137].

${ }^{17}$ Historia et laudes SS. Sabae et Macarii iuniorum e Sicilia auctore Oreste patriarcha Hierosolimitano, graece et latine reddita et adnotationibus illustrata I. Cozza Luzi, Roma, 1893, p. 21.

${ }^{18}$ Vitae Sancti Leonis Lucae abbatis Corilionensis abbati Mulensi in Calabria, in Acta Sanctorum, Martii, I, Parisiis, 1865, p. 98-102. Gli attributi con i quali l'agiografo descrive l'habitat mercuriense «mirae pulchritudinis et amoenae iucunditatis» servono a dare il senso dell'aura di santità in cui era avvolto il monastero che favoriva il raggiungimento della pace interiore, riflettendo la bellezza fisica dei luoghi. Il Bios di San Leoluca di Corleone, è conservato in un codice greco di Palermo di cui esistono copie autentiche a Corleone e a Mazzara. L'opera fu tradotta dal greco al latino e pubblicata dal Gaetani nella sua Vitae Sanctorum siculorum, ex antiquis monumentis pubblicata a Palermo nel 1657 (E. PANDOLFI, Il Beato Leoluca Abate e Mormanno, Castrovillari, 1909, p. 2; più di recente Vita di san Leone Luca da Corleone, cur. M. Stelladoro, Grottaferrata, 1995).

${ }^{19}$ Vita S. Vitalis abbatis Armenti et Rapollae in Italia auctore fere coaevo, in Acta Sanctorum, Martii II, Parisiis, 1865, p. 26-34.

${ }^{20}$ Vita di San Nilo, op. cit. (n. 10), p. 48-49, c. 31.

${ }^{21}$ Ibidem, p. 38-40, c. 22.

${ }^{22}$ Vita di San Nilo Abate fondatore della badia di Grottaferrata, scritta da San Bartolomeo suo discepolo, cur. A. Rocchi, Roma, 1904 , p. 38.

${ }^{23}$ S. NAPOLITANO, Ricordi dell'ascetismo bizantino in Papasidero, in Bollettino della Badia greca di Grottaferrata, n.s., XXX (1976), p. 113- 137: 113-114.

${ }^{24}$ G. GIOVANELLI, L'eparchia monastica del Mercurion, in Bollettino Badia Greca di Grottaferrata, XV (1961), p. 125.
} 
La scelta del territorio non fu casuale ma dovuta alla posizione, prossima al mare e nello stesso tempo nascosta tra i primi contrafforti appenninici, la cui fitta boscaglia la rendeva impenetrabile. E se l'asperità dei luoghi e la mancanza di popolosi centri demici si confaceva all'ideale dei monaci, votati ad un rigido ascetismo, allo studio e al lavoro, conferiva anche un senso di stabilitas e protezione ${ }^{25}$, malgrado le continue incursioni saracene che dal 950 al 1044 destabilizzarono i territori circostanti, a cui si erano sterilmente opposti Ottone I nel 968 e Ottone II nel 981. Il senso di vita comunitario, la variegata composizione sociale e la notevole disponibilità territoriale erano elementi dai quali i monaci traevano il necessario per la sopravvivenza ${ }^{26}$.

Nel X secolo l'area mercuriense appare limitata alla media e bassa valle del Mercure-Lao e isolata dalle importanti arterie di comunicazione, come la via Popilia ${ }^{27}$. La valle del Lao era collegata col versante jonico della regione attraverso un itinerario magno-greco, di cui è difficile rilevare esattamente l'intero tracciato, che probabilmente seguiva il corso del fiume Coscile (l'antico Sybaris) sino a Torre del Mordillo, lambiva il territorio di Altomonte proseguendo per Castrovillari, Morano, Mormanno, Papasidero e Scalea, lungo il corso del $\mathrm{Lao}^{28}$. Gli itinerari degli asceti erano faticosi ed effettuati solitamente a piedi nudi, o eccezionalmente a dorso di mulo.

Non va trascurato infine che i principali artefici della fioritura spirituale dell'eparchia mercuriense furono asceti di origine sicula provenienti dal monastero di San Filippo d'Argira presso Enna, o dai suoi eremi ubicati sull'Etna. Questi giungevano al Mercurio dopo aver risalito la Calabria beneficiati dall'incontro con sant'Elia lo Speleota, o dopo essere stati accolti in fondazioni sotto la sua influenza ${ }^{29}$.

Intorno al 940 anche san Nilo da Rossano raggiunse il Merkourion seguendo il percorso: Thurio, Cassano, Castrovillari, Morano, Mormanno, Laino, fiume Mercure-Lao. Lo stesso percorso da Rossano a Laino, venne effettuato da Ottone II, in un paio di giorni, tra il 31 luglio e il 2 agosto 982, dopo la disfatta di Capo Colonna, sulla direttrice Rossano-Salerno. Lungo la stessa strada si era accampato nell'aprile del 969 anche Ottone I «in confine atque planicie que est inter Cassanum et Petram Sanguinariam $»^{\circ}$. Si tratta, secondo la puntuale ricostruzione di Pietro Dalena, della cosiddetta Via degli eserciti che passava nei pressi della spelonca dell'asceta nel Merkourion. San Nilo, però, percorse probabilmente un sentiero diverso e più rapido, noto soltanto ai locali. Da lì, poi si spostò verso il monastero di San Nazario situato nel Principato salernitano percorrendo un sentiero lungo il fiume Lao (un'antica pista magno-greca), sino a Papasidero. Da qui proseguì per Scalea, per poi risalire la via tirrenica sino a Maratea, Sapri e Camerota da dove, attraverso la via per Celle di Bulgheria-Rocca Gloriosa, raggiunse San Mauro La Bruca arrivando alla sua meta ${ }^{31}$.

Il viaggio si concluse a Valleluce dove, secondo il racconto del Chronicon Casinense, l'abate Potone aveva fatto costruire (probabilmente al tempo di Grimoaldo) una «ecclesiam in honore sancti Angeli» in cui dimorò per qualche tempo «cum aliquot Grecis fratribus» ${ }^{2}$, prima di spostarsi a Grottaferrata. Infatti, come lucidamente sottolinea Vera von Falkenhausen, l'Italia meridionale bizantina era l'ambiente più idoneo per reciproci e approfonditi contatti tra monaci latini e greci non limitati alla semplice 'conoscenza personale' ma indirizzati «alla comprensione delle diversità e delle somiglianze». Rimanevano alcune zone d'ombra, indizio che la convivenza era spesso basata su una pace precaria: lo stesso Nilo, ad esempio, nonostante avesse trovato in Aligerno di Montecassino un fedele amico, si sentiva ugualmente dipendente da una comunità monastica straniera, tanto da declinare la richiesta di Adalberto di Praga il quale, non soddisfatto della vita a Montecassino, chiedeva di seguirlo e, alla morte di Aligerno quando l'ultima «scintilla dello spirito di fratellanza ecumenica si spense», decise di lasciare Valleluce ${ }^{33}$.

\section{LA RINASCITA CASSINESE}

In un contesto segnato dalla pervasiva presenza monastica italo greca, tuttavia, dalla seconda metà del IX secolo iniziò a registrarsi la presenza di qualche insediamento dipendente dalle grandi abbazie latine (Montecassino, San Vincenzo al Volturno), soprattutto nel quadrante settentrionale della regione rimasta a lungo sotto la dominazione longobarda, sede di importanti circoscrizioni gastaldali34.

\footnotetext{
${ }_{25}$ B. CAPPELLI, Medioevo bizantino nel Mezzogiorno d'Italia ed altri saggi di storia e d'arte medievale, Castrovillari, 1993, p. 65.

${ }^{26}$ «Padre, andiamo nel luogo dove era il frumento, e vedrai che non ho mentito alla tua reverenza. Subito quel famoso santo si recò insieme e avendo iniziato a frugare con le proprie mani (di cosa miracolosa, di dono di Cristo!) trovò il vaso miracolosamente pieno di frumento, che proprio poco prima era vuoto. Allora quello comandò che fossero aperte le giare che erano intorno, avendolo fatto, le altre due furono trovate piene di frumento» [Historia et laudes SS. Sabae et Macarii, op. cit. (n. 17), p. 26]. E ancora: «Si apre fosca e profonda la gola intagliata nelle selvose montagne di Papasidero da cui prorompe, nella piana, il Lao come da un'immane e favolosa bocca di drago. Di fronte e al di là dell'alveo di questo fiume si sollevano in primo piano le colline, pingui di vigneti, frumento ed olivi, che ascendono ai lussureggianti castagneti di Trémoli» (B. CAPPELLI, Il monachesimo basiliano ai confini calabro-lucani, Napoli, 1963, p. 201).

${ }^{27}$ B. CAPPELLI, Medioevo bizantino nel Mezzogiorno d'Italia, op. cit. (n. 25), p. 71-72.

${ }^{28}$ P. DALENA, Dagli itinera ai percorsi. Viaggiare nel Mezzogiorno medievale, Bari, 2003, p. 115.

${ }^{29}$ F. BURGARELLA, L'eparchia di Mercurio, op. cit. (n. 16), p. 71.

${ }^{30}$ Conradi I. Heinrici I. et Ottonis. Diplomata, in Monumenta Germaniae Historica (= MGH), Diplomatum regum et imperatorum Germaniae, I, Hannoverae, 1884 , p. 508-510, doc. 371.

${ }^{31}$ P. DALENA, Dagli itinera ai percorsi, op. cit. (n. 28), p. 205-208.

${ }^{32}$ Chronica monasterii Casinensis, edid. H. Hoffmann, MGH, Scriptores, XXXIV, Hannover 1980, p. 201.

33 V. VON FALKENHAUSEN, I monasteri greci dell'Italia meridionale e della Sicilia dopo l'avvento dei Normanni: continuità e mutamenti, in Il passaggio dal dominio bizantino allo stato normanno nell'Italia meridionale, Atti del II Convegno internazionale sulla civiltà rupestre medievale nel Mezzogiorno d'Italia (Taranto-Mottola, 31 ottobre - 4 novembre 1973), Taranto, 1977, p. 197-219.

34 Nell'839-840 Siconolfo impossessatosi della città di Salerno, dopo aver sconfitto Radelchi a stento scampato alla morte, intraprese la conquista dei territori ad est di Salerno, quelli della Valle del Crati (Cosenza, Cassano e Bisignano) che oscillavano tra la dipendenza formale da Benevento e le ingerenze bizantine. Un decennio dopo il principe di Benevento, Radelchi I divise i propri territori con Siconolfo: ricadevano sotto la dominazione di quest'ultimo
} 
Se il territorio di Malvito, ad esempio, ricadeva ancora agli inizi del $\mathrm{X}$ secolo nella sfera di interesse patrimoniale dell'abbazia benedettina di San Vincenzo al Volturno, come dimostra un contratto di permuta in cui Goldeperto abate di San Vincenzo cede a Yselgrimo vescovo di Cosenza i beni controllati dalla sua abbazia nel territorio di Malvito, in cambio dei beni episcopali in Visuniano (Bisignano) cum omni sua pertinencia ${ }^{35}$; già dai decenni precedenti, sono ben documentati i rapporti tra l'abbazia di Montecassino e il monastero di Santa Maria di Cosenza, sua dipendenza, dotato di numerosi beni immobiliari ${ }^{36}$.

Montecassino, infatti, tra VIII e IX secolo (epoca segnata dalla prima ricostruzione nel 717, dopo la distruzione longobarda nel 577, e dalla successiva distruzione saracena nell'883), aveva accumulato un ingente complesso di beni patrimoniali i cui confini si estendevano dall'Italia settentrionale alla Sicilia, dalla Spagna, alla Francia fino alla Dalmazia e alla Turchia ${ }^{37}$.

Alla base di questo processo formativo, che per le terre campane e basso laziali portò all'uso della denominazione di Terra Sancti Benedicti ${ }^{8}$, vi erano motivi di ordine spirituale perché l'abbazia era diventata frontiera della nuova 'latinizzazione' del Mezzogiorno e culla del monachesimo occidentale; ma anche politici perché la geografia dei territori in cui sorse trasformò il Mezzogiorno nello scenario delle grandi contese politiche e militari del medioevo, tra longobardi e bizantini, arabi e normanni; si trattava insomma di una vasta signoria "protetta dalle più ampie immunità, che ne facevano quasi uno stato del tutto autonomo, sapientemente organizzato e con saggezza amministrato dai monaci benedettini» 39

Oltre i confini della Terra Sancti Benedicti era attiva una rete di dipendenze a cui apparteneva anche il monastero cosentino, probabilmente di origine italo-greca, passato sotto il controllo di Montecassino tra l'858 e l'867 quando ne fu ordinata la ricostruzione. E, il 18 aprile dell'863 l'abate Basaccio ne confermava «omnia iura, privilegia, bona et ecclesia». Tale monastero rappresentava un importante avamposto latino nella Valle del Crati, in un territorio tradizionalmente popolato da enclaves monastiche italo greche. La dipendenza benedettina, tuttavia, non sembrava porsi in antagonismo con le realtà italo greche e anzi, specie dal $\mathrm{X}$ secolo in poi, le fonti sembrano testimoniare una osmosi culturale, e cultuale, tra le due realtà ${ }^{40}$.

Ubicato nella parte meridionale della città di Cosenza, sotto il castello, di fronte alla cattedrale, il 22 maggio $882 \mathrm{fu}$ esentato da Giovanni VIII dal pagamento dei censi (l'anno prima che Montecassino subisse l'onta della seconda distruzione) insieme con altri tre monasteri femminili dell'Italia meridionale ricadenti sotto il protettorato cassinese ${ }^{41}$. Qualcosa di più preciso sulla consistenza patrimoniale del monastero cosentino si ricava, invece, da un documento del marzo 899 in cui si fa riferimento a curtis e casalibus nei quali vivevano servis e ancillis che prestavano servizi lavorativi nelle terre ubicate anche fuori regione. Quest'ultimo inciso rivela la vivacità economica delle pertinenze cassinesi e il loro fondamentale apporto all'opera di dissodamento e messa a coltura di nuovi territori ${ }^{42}$. E anche la riconferma dei privilegi, ad opera dell'abate Aligerno, sembra accreditare l'intraprendenza delle monache calabresi che estendevano la loro influenza in non meglio precisati «loca in finibus Beneventanis et in Apuliis et Kalabricis», poi trasformati in nuove dipendenze cassinesi ${ }^{43}$.

Questa politica di rinnovo delle concessioni si segue per tutto il X secolo come dimostrano i documenti dal 944 al 989 e quelli successivi dal 1014 al $1067^{44}$. Invece nel privilegio di Urbano II (27 marzo 1097) assieme al monastero cosentino compaiono, tra le dipendenze cassinesi in Calabria, anche la «cella Sancte Anastasie, Sancti Nicolai in Salectano, Sancte Marie in Tropea, Sancte Heuphimie in Marchia» e Cetraro ${ }^{45}$.

i seguenti castaldata: Tarantus, Latinianus, Cassanus, Cusentia, Laynus, Lucania, Conscia, Muntella, Rota, Salernus, Sarnus, Cymitirium, Furcule, Capua, Tianus, Sora e metà gastaldato Aceruntinus, esattamente la parte congiunta con il Latiniano et Consciam [J.-M. MARTIN, Guerre, accords et frontières en Italie méridionale pendant le haut Moyen Âge. Pacta de Liburia, Divisio pincipatus Beneventani et autres actes, Rome, 2005 (Sources et documents d'Historie du Moyen Âge, 7), p. 201-217: 205, doc. 4].

${ }_{35}$ Chronicon Vulturnense del monaco Giovanni, cur. V. Federici, II, Roma, 1925, p. 39; III, p. 149, doc. 48.

${ }^{36}$ La prima notizia risale al 18 aprile 863: Abbazia di Montecassino. I regesti dell'archivio, cur. T. Leccisotti, I, Roma, 1964 (Pubblicazioni degli Archivi di Stato, 54), p. 223-224, n. 6.

${ }^{37}$ Sull'estensione complessiva del patrimonio fuori dall'Italia si vd. la bolla di Alessandro III del 7 novembre 1159 in Abbazia di Montecassino. I regesti, op. cit. (n. 36), p. 9 e E. GATTOLA, Historia abbatiae Cassinensis per saeculorum seriem, Venetiis, 1733, p. 338. L'elenco completo dei possedimenti è invece nel diploma di Lotario III del 10 ottobre 1137, confermato da Enrico VI il 21 maggio 1191 in Abbazia di Montecassino. I regesti, II, p. 33, 46, 54 e E. GATTOLA, Ad historiam abbatiae Cassinensis accessiones, Venetiis, 1734, p. 250, 269. Su quest'ultimo grava un pesante sospetto di falsificazionead opera di Pietro Diacono. ${ }^{38}$ Questa denominazione appare per la prima volta in un documento del 982 contenente un atto di vendita del territorio di Aquino. Fu poi ripresa in un diploma di Ottone III col quale l'imperatore di casa Sassonia cedeva Pontecorvo e il suo territorio a Giovanni console di Gaeta nel 999 (Codex diplomaticus Cajetanus, editus cura et studio monachorum S. Benedicti archicoenobii Montis Casini, I, Montecassino, 1887, p. 193-195, n. CII) e successivamente venne utilizzato dagli annalisti cassinesi nel $1045 \mathrm{i}$ quali annotavano che in quell'anno «Richerius Abbas eiecit Normannos de Terre Sancti Benedicti» (Annales Casinenses ex annalibus Montis Casini antiquis et continuatis excerpti inde ab a. 1000 usque ad a. 1089, edid. W. Smidt, MGH, Scriptores, XXX, Leipzig, 1934 p. 1414-1415).

39 L. FABIANI, La terra di S. Benedetto. Studio storico-giuridico sull'abbazia di Montecassino dall'VIII al XIII secolo, I, Montecassino, 1968 , p. 3-62.

$4^{4}$ Supra, n. 36.

${ }^{41}$ E. GATTOLA, Historia abbatiae, op. cit. (n. 37), p. 63; P.F. KEHR, Italia Pontificia, VIII, Regnum Normannorum - Campania, Berlin, 1934, p. 126, n. 37 (d'ora in poi: KEHR)

${ }^{42}$ KEHR, VIII, p. 127, n. 41, ID., Le bolle pontificie anteriori al 1198, in Miscellanea Cassinese, II (1899), p. 1-90: 26 , n. 2.

${ }^{43}$ P.F. KERH, Bolle pontificie anteriori al 1199, op. cit. (n. 42), p. 30-32.

${ }^{44} \mathrm{Si}$ vd. la recente edizione del Registrum Petri Diaconi (Montescassino, archivio dell'abbazia, reg. 3); curr. J.-M. Martin et alii, I, Rome, 2015 (Sources et documents publiés par l'Ėcole française de Rome, 4), p. 69-72, n. 8; 86-100, n. 13-16; 105-108, n. 18; 113-120, n. 20-21; 129-152, n. 26-29.

${ }^{45} \mathrm{Ibidem}$, p. 167-172, n. 36; poi confermati da Pasquale II il 17 marzo 1105 a Oderisio I abate (ivi, p. 184-189, n. 40). Il locus Cetraro e il suo porto fu offerto all'abate Desiderio da Sichelgaita, moglie di Roberto il Guiscardo, che lo aveva ricevuto in dote dal marito (morgengabe) (ivi, III, p. 1187-1189 n. 421, gennaio-aprile 1086). Nell'agosto del 1090, invece, il duca Ruggero Borsa conferma a Oderisio II le concessioni fatte dal padre e dalla madre Sichelgaita, 
Ciò dimostra che con l'arrivo dei normanni l'archicenobio cassinense espande la sua influenza nella regione, controllando nuovi territori e fondazioni religiose, gli opifici ad esse connessi e il porto di Cetraro con la relativa rendita sul pescato $^{46}$.

\section{DIPENDENZE CAVENSI}

Dalla metà dell'XI secolo, invece, è possibile seguire con buona continuità le relazioni tra la Badia di Cava e alcuni monasteri italo greci della Calabria settentrionale. Le recenti ricerche di Barbara Visentin ${ }^{47}$, che muovono dal lavoro di Leone Mattei Cerasoli ${ }^{4}$ e dai più recenti studi di Giovanni Vitolo $^{49}$ sulle dipendenze cavensi, hanno dimostrato che attraverso il vallum Diani si realizzò una profonda osmosi culturale e religiosa, con l'apertura di un corridoio geografico attraverso cui Cava riusci ad estendere la sua influenza sulle aree lucane e su quelle dell'alta Calabria ${ }^{50}$.

Già agli inizi dell'XI secolo gli abati cavensi avevano realizzato le premesse di questa dinamica stabilendo qualche avamposto nel Cilento, ma solo tra il 1079 e il 1083, incamerando il monastero di San Biagio di Sartiano, riuscirono ad avviare pienamente il processo di colonizzazione spirituale della regione estendendo l'azione nella Valle del Sinni e a sud del Pollino ${ }^{51}$. Era questa la cosiddetta seconda fase di propagazione della congregazione cavense che - come ha avuto modo di sottolineare Giovanni Vitolo - almeno per la Puglia e il Cilento non era inserita nel progetto normanno di latinizzazione delle strutture ecclesiastiche e monastiche, ma in un più dinamico orizzonte politico e spirituale che accanto al calcolo politico e alla forza della spiritualità dei cavensi, includeva certamente l'azione riformatrice di vescovi e pontefici ${ }^{52}$.

Profondamente diverso il contesto della Basilicata meridionale e della Calabria settentrionale nel quale accanto ad una forte componente latina e ad ambienti rurali longobardizzati si nota una preponderanza dell'elemento monastico italo-greco che, come è stato ampiamente messo in evidenza precedentemente, costituiva un forte catalizzatore sociale per gli uomini del tempo.

I cavensi, invece, sostenuti dai Chiaromonte, famiglia di origine normanna stanziatasi nella parte meridionale della Basilicata, iniziarono una lenta opera di colonizzazione spirituale delle zone del Latiniano ricevendo dal conte Ugo il monastero di S. Maria di Kirzosimo (21 novembre 1088) «cum hominibus et aliis pertinentiis suis et cellis, que grece dicitur metochia»53. Il monastero di Cersosimo era stato fondato tra la fine del X e i primi anni dell'XI secolo al centro di un territorio dove si registrò una cospicua presenza di monaci italo greci migrati dalla Sicilia e dalla Calabria meridionale, al confine tra l'eparchia del Latiniano e quella del Mercurio. Al momento della fondazione, emerge dalla documentazione superstite, furono le stesse autorità bizantine a favorire l'insediamento monastico per trasformarlo in cerniera tra gli insediamenti italo greci calabresi e quelli salentini ${ }^{44}$.

Ma già due anni prima (novembre 1086), Ugo signore normanno di Avena (frazione di Papasidero, Cs) con la moglie Emma e il figlio Ugo, avevano donato a Pietro abate cavense ben tre monasteri italo greci nell'area di Laino Castello: Sancti Iohannis in loco Layta, prossimo al Kastron Mercurio, San Nicola de Padule e San Simeone in loco pertinentiis de castello Montesano55.

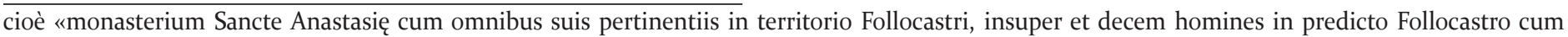
omnibus familiis et possessionibus ac substantiis eorum»; «et monasterium Sancti Nykolai in territorio Bisinianensi» (ivi, p. 1402-1404, n. 512). Qualche anno più tardi (1094, o forse 1102, la datazione è incerta), Rocca figlia del conte Drogone, col consenso del conte di Sicilia, donò anche undici famiglie di villani dimoranti in vico Sallitano (ivi, p. 1412-1413, n. 516); e, tra il 1097 e l'anno successivo, offre a Montecassino e a Ihoannes Bellus, preposito di S. Nicola de Sallittano, uomini e terre in vico Sallittano (ivi, p. 1416-1419, n. 518) e nel febbraio 1104 (o forse 1105) dona all'abate Oderisio I e al rector di S. Benedetto di Cetraro, Sasso, metà della chiesa di S. Pietro ad Ferolitum, di quella di S. Demetrio e di tutti i suoi beni tra cui un porto non meglio identificato (ivi, p. 1414-1415, n. 517). Infine, una terra «Sancti Nicolai de Sallitano» è menzionata in un documento col quale Guglielmo II, nel dicembre 1166, concede al monastero della Sambucina cinquanta some siciliane di terra regia di cui indica i confini [A. PRATESI, Carte latine provenienti dall'Archivio Aldobrandini, Città del Vaticano, 1958 (Studi e testi, 197), p. 58-60, n. 22].

${ }^{46}$ C.R. COSENZA, La donazione di Cetraro della duchessa Sikelgaita alla abbazia di Montecassino e lontana incidenza del monachesimo benedettino nella realtà economica e sociale del Tirreno cosentino, in Atti del $7^{\circ}$ Congresso internazionale di studi sull'alto medioevo, II, Spoleto, 1982, p. 647-655; P. DE LEO, Nel dominio dell'abate: un feudo cassinese in Calabria, in ID., Mezzogiorno medievale: istituzioni, società, mentalità, Soveria Mannelli, 1984, p. 67-109.

${ }^{47}$ B. VISENTIN, Fondazioni cavensi nell'Italia meridionale (secoli XI-XV), Battipaglia, 2012; EAD., Il monachesimo dei grandi spazi aperti. I cavensi in Lucania, Puglia e Calabria (secc. XI-XII), in Riforma della Chiesa, esperienze monastiche e poteri locali. La Badia di Cava nei secoli XI-XII, Atti del convegno internazionale di studi (Badia di Cava, 15-17 settembre 2011), cur. G. Vitolo et alii, Firenze, 2014, p. 135-148; EAD., Percorsi monastici nel Mezzogiorno medievale. La congregazione di Cava, 2 voll., Battipaglia, 2015. Si vd. anche per un quadro complessivo il sempre valido lavoro di P. GUILLAUME, Essai historique sur l'Abbaye de Cava d'après des documents inédits, Cava dei Tirreni, 1877.

${ }^{48}$ L. MATTEI-CERASOLI, La Badia di Cava e i monasteri della Calabria superiore, in Archivio storico per la Calabria e la Lucania, 8 (1938), p. 167-182, 265285; 9 (1939), p. 279-318.

${ }^{49}$ G. VITOLO, La latinizzazione dei monasteri italo-greci del Mezzogiorno medievale. L'esempio di S. Nicola di Gallocanta presso Salerno, in S. LEONE, ID., Minima Cavensia. Studi in margine al IX volume del Codex Diplomaticus Cavensis, Salerno, 1983, p. 75-92; ID., Insediamenti Cavensi in Puglia, in L'esperienza monastica benedettina e la Puglia, cur. C.D. Fonseca, II, Galatina, 1984, p. 4-166; ID., Il monachesimo latino nell'Italia meridionale (secc. XI-XII), in Benedictina, 35 (1988), p. 543-553; ID., I caratteri del monachesimo cavense. L'esperienza di un millennio, in Riforma della Chiesa, op. cit. (n. 47), p. 3-12.

${ }^{50}$ Questo era favorito dal particolare contesto religioso in cui operavano sia la curia romana che l'episcopato locale per il rilancio dei principi della riforma e per ripristinare le attività pastorali; mentre i Normanni realizzavano il complessivo riassetto politico del Mezzogiorno. A tutto ciò, dopo la normalizzazione della conquista, si aggiunse la necessità da parte dei signori di 'ammantare' il loro potere di responsabilità che derivavano dal ruolo di mecenati-protettori di chiese e monasteri [G. VITOLO, I caratteri del monachesimo cavense, op. cit. (n. 49), p. 5].

${ }^{51}$ V. RAMSEYER, Vescovi e monasteri nei secoli XI-XII, in Riforma della Chiesa, op. cit. (op. 49), p. 40.

${ }^{52}$ G. VITOLO, Insediamenti cavensi in Puglia, op. cit. (n. 49), p. 10-12.

${ }_{53}$ Codex Diplomaticus Cavensis, XII, 1086-109o, curr. G. Vitolo et alii, Battipaglia, 2015, p. 240-241, n. 9o; L. MATTEI CERASOLI, La badia di Cava e i monasteri greci, op. cit. (n. 48), p. 275-276.

54 B. VISENTIN, Fondazioni cavensi, op. cit. (n. 47), p. 263-265

${ }_{55}$ Codex Diplomaticus Cavensis, XII, op. cit. (n. 53), p. 91-93, n. 35; L. MATTEI CERASOLI, La badia di Cava e i monasteri greci, op. cit. (n. 48), p. 176-177. È importante far rilevare - come sottolineano anche gli editori - che i tre monasteri non compaiono più nella documentazione cavense, né sono stati 
La comunità di Cava, inoltre, deteneva il monastero di Sancti Nicolai de Cofina a Oriolo, la cui donazione risale al 1132, anche se lo controllavano dalla fine dell'XI secolo ${ }^{56}$; e sempre lungo quella linea di confine entrò tra le dipendenze di Cava il monastero di San Pietro in Brahalla la cui donazione è attestata da un documento redatto nel $1114^{57}$. Qui, probabilmente, i cavensi furono favoriti dai Grantmesnil, una nobile famiglia normanna stanziatasi tra Calabria e Lucania, come risulta dalla cartula donationis successiva (1117) con la quale la badia riceve da Mabilia, figlia del Guiscardo, contessa di Oriolo e vedova di Guglielmo Grantmesnil, e dal figlio Guglielmo la chiesa «cum casali et pertinentiis», vigne, campi coltivati, boschi e case, ma anche l'acquatico per abbeverare gli animali da soma della Badia e degli uomini del casale di San Pietro ${ }^{5^{8}}$. Esso dimostra inoltre la tuitio esercitata dalla famiglia del Guiscardo nei confronti dei cavensi, elemento quest'ultimo che potrebbe essere interpretato come volontà normanna nell'utilizzare anche il cenobio quale vettore della latinizzazione in quest'area, al contrario di quanto avvenne per le fondazioni pugliesis9.

E se la formazione del patrimonio cavense deve essere attribuita all'intuizione dei due Guaimario (terzo e quarto) principi di Salerno, è altrettanto vero che l'esperienza politica longobarda era oramai al termine e solo la lungimiranza degli abati riuscì a ridisegnare il progetto espansionistico, inserendolo negli spazi della nuova conquista normanna, così da estendere non soltanto gli ambiti territoriali di influenza badiale, ma di ampliarne anche l'orizzonte economico e culturale, specie dopo il 1070 quando Cava si apprestava a diventare una signoria fondiaria vera e propria, almeno nel Cilento e nei possedimenti della Calabria settentrionale dove «i suoi interlocutori sembrano non disporre di un patrimonio fondiario esteso, che potesse assicurare protezione e fortuna personale ${ }^{60}$.

\section{DALLA CONQUISTA NORMANNA ALLA LATINIZZAZIONE}

L'equilibrio raggiunto in Calabria tra la Chiesa greca e quella latina, incrinato dapprima dallo scisma di Michele Cerulario (1054) che divise le due Chiese sancendo il fallimento del rapporto tra il papato e Bisanzio ${ }^{61}$, fu definitivamente compromesso dagli accordi di Melfi del 1059 attraverso i quali la sede apostolica legittimava la conquista della regione ultimata, secondo il racconto del Malaterra, con la presa di Reggio Calabria ${ }^{62}$. Con il patto sottoscritto nel 1059 la nuova funzione militare del Guiscardo (acclamato dux dalle truppe sul campo) venne mutata da Nicolò II, nel concilio di Melfi ${ }^{63}$, in esercizio effettivo del potere mentre il normanno giurava di riportare le chiese greche di Calabria all'obbedienza alla sede apostolica ${ }^{6}$. Secondo la formula, Roberto il Guiscardo prometteva al pontefice che «ad confirmationem traditionis et ad recognitionem fidelitatis, (...), promitto me annualiter pro uno quoque jugo bonum pensionem, scilicet duodecim denarios papiensis monete, persoluturum beato Petrus et tibi domino meo Nicolao pape et omnibus successoribus tuis, aut tuis aut tuorum successorum nuntiis omnes ecclesiasque in mea constituit dominatione cum earum possessionibus» ${ }^{65}$. La stessa fonte, tuttavia, riproduce un secondo giuramento nel quale era previsto che il normanno corresse in aiuto del pontefice in caso di necessità, impegnandosi altresì a «terramque Sancti Petri et principatus nec invadere nec acquirere queram nec

oggetto di contesa con altri enti religiosi, perché trattandosi di una falsificazione è probabile che nella donazione originale fossero menzionati soltanto la chiesa di San Giovanni e il monastero dei SS. Quaranta (da identificare con S. Simeone a Laino Castello), come si evince da un successivo privilegio di Urbano II (1089). Quest'ultimo elemento permette di datare la falsificazione tra il 1089 e il 110o allorché col privilegio di Pasquale II anche il monastero di S. Nicola compare regolarmente tra le dipendenze cavensi [B. VISENTIN, Fondazioni cavensi, op. cit. (n. 47), p. 316-318; EAD., Percorsi monastici, II, op. cit. (n. 47), p. 203-205].

${ }^{56}$ F. TRINCHERA, Syllabus graecarum membranarum, Napoli, 1865, p. 148-150, n. CXII; L. MATTEI CERASOLI, La badia di Cava e i monasteri greci, op. cit. (n. 48), p. 281; B. VISENTIN, Fondazioni cavensi, op. cit. (n. 47), p. 319-320; EAD., Percorsi monastici, II, op. cit. (n. 47), p. 195-197.

${ }^{57}$ F. TRINCHERA, Sillabus, op. cit. (n. 56), p. 99-100, n. LXXVI; L. MATTEI CERASOLI, La badia di cava e i monasteri greci, op. cit. (n. 48), p. 268; B. VISENTIN, Fondazioni cavensi, op. cit. (n. 47), p. 322-324; EAD., Percorsi monastici, II, op. cit. (n. 47), p. 198-202.

${ }_{58}^{8}$ F. TRINCHERA, Sillabus, op. cit. (n. 56), p. 108-110, n. LXXXIII; P.M. TROPEANO, Codice Diplomatico Verginiano, II, 1102-1132, Montevergine, 1978, p. 133-139, n. 132; B. VISENTIN, Fondazioni cavensi, op. cit. (n. 47), p. 322-323; EAD., Percorsi monastici, II, op. cit. (n. 47), p. 199.

59 Supra, n. 52.

${ }^{60}$ B. VISENTIN, Il monachesimo dei grandi spazi aperti, op. cit. (n. 47), p. 139.

${ }^{61}$ Sullo scisma si vd. E. BRÉHIER, Le schisme oriental du XIe siècle, Paris, 1899; M. JUGIE, Le schisme de Michel Cérulaire, in Echos d'Orient, 37 (1937), p. 440-473; V. GRUMEL, Les préliminaires du schisme de Michel Cérulaire ou la question romaine avant 1054, in Revue des études byzantines, 10, 1 (1952), p. 5-23. ${ }^{62}$ P. DALENA, Calabria medievale. Ambiente e istituzioni (secc. XI-XV), Bari, 2015, p. 143-151: 148-149; si vd. anche C. HERVÉ-COMMEREUC, Les normands en Calabre, in Les Normands en Méditerranée dans le sillage del Tancrède, Colloque de Cerisy-La-Salle (24-27 septembre 1992), curr. P. Bouet, F. Neveaux, Caen, 1994, p. 77-87.

${ }^{63}$ GOFREDO MALATERRA, De rebus gestis Rogerii Calabriae et Siciliae Comitis et Roberti Guiscardi ducis fratris eius, ed. E. Pontieri, Bologna, 1928 (Rerum italicarum scriptores, V), p. 23, c. XXXV. Nota il Pontieri che né Malaterra, né Amato di Montecassino fanno diretto riferimento al concilio di Melfi e all'investitura del Guiscardo. Tuttavia il concorso di altre fonti cronachistiche, quali Leone Ostienense e Romualdo Salernitano, sembrano convalidare tale ipotesi. Da esse si ricava, infatti, che soltanto dopo la presa di Reggio il Guiscardo assunse il titolo di Dux.

${ }^{64}$ Sul problema della rilatinizzazione vd. supra nota n. 5 e anche P. DALENA, Istituzioni religiose e quadri ambientali nel Mezzogiorno medievale, Cosenza, 1997, p. 78; ID., La conquista normanna e la latinizzazione della Chiesa in Calabria, in Quaderni Lametini, 29 (1994), p. 17-34; ID., La Calabria in età normanna: aspetti e problemi, in Mezzogiorno-Federico II-Mezzogiorno, Atti del convegno internazionale di studio promosso dall'Istituto internazionale di studi federiciani, CNR, (Potenza-Avigliana-Castel Lagopesole-Melfi, 18-23 ottobre 1994), I, Roma, 1999, p. 343-379; S. CARUSO, Politica "gregoriana", latinizzazione della religiosità bizantina in Italia meridionale, isole di resistenza greca nel Mezzogiorno d'Italia tra XI e XII secolo, in Cristianità d'Occidente e cristianità d'Oriente (secoli VI-XI), I, LI Settimana di studio del Centro italiano di studi sull'alto medioevo (Spoleto, 24-30 aprile 2003), Spoleto, 2004, p. 463-547; P. DALENA, Calabria medievale, op. cit. (n. 62), p. 158-163. Gli impegni di Melfi vennero rinnovati a Ceprano (1080), cfr. P. DALENA, Urbano II e la Calabria, in Bollettino storico della Basilicata, IX (1993), p. 45-65: 45-46.

${ }^{65}$ Liber Censuum Romanae Ecclesiae, edit. P. Fabre, I, Paris, 1889, p. 421-422, n. 162. 
etiam depredari presumam»; rimettendo in possesso del papa «omne quoque ecclesiasque in mea consistunt dominatione cum earum possessionibus» ${ }^{66}$.

Che si tratti di un giuramento vassallatico non sembrano esservi dubbi e anzi, secondo alcuni studiosi, funse da modello per successivi giuramenti feudali ${ }^{67}$. Di diverso avviso Vincenzo d'Alessandro, il quale ritiene che pur trattandosi di un giuramento di fedeltà, non si riferisca esplicitamente alla fedeltà vassallatica; perché «a Melfi Nicolò II riconosceva per la Chiesa il passaggio in mano ai normanni dei territori meridionali, alla cui perdita non aveva potuto opporsi Bisanzio e lo stesso papato». Analogamente riconosceva e legittimava il titolo assunto da Roberto, chiedendo la restituzione delle chiese e il ripristino delle prerogative ecclesiastiche. I normanni, allora, avrebbero più semplicemente giurato obbedienza e lealtà ai pontefici, da cui avrebbe avuto origine soltanto il potere ducale. In altri termini il papato, non intendendo confliggere con l'imperatore d'Occidente, attraverso l'accordo melfitano recuperava il controllo delle chiese rimaste lungo tempo sotto il controllo di Bisanzio ${ }^{68}$.

D’Alessandro, riprendendo le tesi di Jules Gay e Giuseppe De Blasiis, sembra non considerare però quanto riportato da Guglielmo di Puglia secondo cui dopo la conferma del titolo ducale Niccolò investì Roberto «unde sibi Calaber concessus et Appulus omnis / est locus, et Latio patriae dominatio gentis» ${ }^{69}$; e ancora non tiene conto dell'atteggiamento normanno dopo la Battaglia di Benevento in cui fu sconfitto e imprigionato papa Leone IX (1053) e quello dei pontefici successivi, che cercarono di esercitare uno stringente controllo sulle politiche meridionali.

I normanni si erano quindi impegnati, da un lato nella riorganizzazione dei distretti carismatici, dall'altro nel pa- trocinio di nuove fondazioni monastiche benedettine che sarebbero divenute centri di irradiazione del nuovo culto latino e fondamentali unità produttive per il rilancio economico della regione ${ }^{70}$; favorendo quindi sia l'esigenza pontificia di marginalizzare il rito greco; che il processo di unificazione della conquista con la conseguente affermazione politica e sociale sui territori bizantini ${ }^{71}$.

Del resto la particolare attenzione del papato all'Italia meridionale e alla Sicilia era nota da prima del concilio di Melfi come dimostrano, ad esempio, la nomina arcivescovile di Umberto di Silva Candida a Palermo, in Sicilia da parte di Leone IX nel $1050^{72}$; o la celebrazione dei sinodi romani (1049) sulla questione delle decime e quella dei concili di Siponto e Salerno (1050) contro i vescovi simoniaci73.

La Chiesa italo greca, quindi, dopo aver cercato vie di integrazione con le compagini latine e aver raggiunto la sua massima espansione, subì una importante battuta d'arresto anche se rimasero attivi alcuni centri monastici e diocesi per lungo tempo. Infatti gli Altavilla, attenti alla composizione etnica della popolazione, per evitare conflitti e sommosse, avallarono la nomina di qualche vescovo greco in zone fortemente grecizzate e furono particolarmente munifici nei confronti dei monasteri con cospicue donazioni di uomini e beni, come nel caso del monastero di San Pancrazio di Briatico (1062), dell'abbazia greca di San Nicola di Drosi (1083) e del monastero di San Nicodemo da Grutaria (1092)74.

Fu forse questa la forza di una conquista non programmata che portò alla rapida unificazione dei territori della Calabria settentrionale e meridionale, cui fu data una nuova identità politica, con l'introduzione del modello feudale normanno ${ }^{75}$, e religiosa, con l'avvicendamento dei presuli greci con ordinari latini ${ }^{76}$; la fondazione di una serie di mo-

\footnotetext{
${ }^{66}$ Ibidem, p. 422, n. 163.

${ }^{67}$ W. ULLMANN, The Growth of Papalgouvernement in Middle Ages. A study in the ideological relation of clerical to lay powers, London, $1965, \mathrm{p} .337$.

${ }^{68}$ V. D'ALESSANDRO, Storiografia e politica nell'Italia normanna, Napoli, 1978 (Nuovo medioevo, 3), p. 133.

${ }^{69}$ GUILLAUME DE POUILLE, Le geste de Robert Guiscard, cur. M. Mathieu, II, Palermo, 1961 (Istituto di studi bizantini e neoellenici. Testi e monumenti, 4), vv. 404-405, p. 154.

$7^{70}$ Parlo diffusamente del fenomeno e delle fondazioni monasteriali in I feudi dell'Abbazia benedettina di Sant'Eufemia in un diploma federiciano del 1240 , in P. Dalena e B. Saitta (a cura di), Enrico Pispisa. Dalla storia alla memoria, Bari, 2014, p. 123-146.

${ }^{71}$ P. DALENA, Istituzioni religiose, op. cit. (n. 64), p. 77.

${ }^{72}$ P.F. KEHR, Italia pontificia, X, Calabria-insulae, edid. D. Girgensohn, W. Holtzmann, Turici, 1975, p. 186, n. 73.

73 AMATO DI MONTECASSINO, Storia de' Normanni, cur. V. De Bartholomaeis, Roma, 1935 (Fonti per la Storia d'Italia, 76), p. 128-130; il viaggio del pontefice nell'Italia meridionale è menzionato negli Annales Beneventani, MGH, Scriptores, III, edid. G.H. Pertz, Hannoverae, 1839, p. 179; LEONE OSTIENSE, Chronica monasterii casinensis, MGH, Scriptores, VII, edid. G.H. Pertz, Hannoverae, 1846, p. 683-784. Per il pontificato di Leone IX si vd. M.G. AGOSTINO, Il primato della sede di Roma in Leone IX (1049-1054). Studio dei testi nella controversia greco-romana nel periodo gregoriano, Cinisello Balsamo, 2008. Sui motivi si vd. F. CHALANDON, Histoire de la domination normande en Italie et en Sicile, I, Paris, 1907, p. 126-127.

${ }^{74}$ Per il monastero di San Pacrazio di Briatico si vd. C. ROGNONI, Les actes privés grecs de l'archivo ducal de Medinaceli (Tolèede), I. Les monastères de Saint-Pancrace de Briatico, de Saint-Philippe-de-Bojôannès et de Saint-Nicolas-de-Dorsi (Calabre, XI ${ }^{e}$-XII ${ }^{e}$ siècles), Paris, 2004, p. 115-118, n. 11; per quanto riguarda San Nicola di Droso si vd. Documenti latini e greci del conte Ruggero I di Calabria e Sicilia, Edizione critica a cura di J. Becker, Roma, 2013 (Ricerche dell'Istituto storico germanico di Roma, 9), p. 53-55, n. 6 e per San Nicodemo da Grutaria, Ibidem, p. 112-113, n. 22. Al contrario, però, alcune diocesi di rito greco venivano soppresse ed i territori accorpati a quelle di rito latino come nel caso di Vibona e di Tauriana che nell'ottobre 1086 furono sottomesse alla nuova sede vescovile di Mileto (Ibidem, p. 64-73, n. 10; si tratta di un documento interpolato ma non falso e per la ricostruzione del contesto storico si rimanda a J. BECKER, Graf Roger I. von Sizilien. Wegbereiter des normannischen Königreichs, Tübingen, 2008 (Bibliothek des Deutschen Historischen Instituts in Rom, 117), p. 162-166 e V. VON FALKENHAUSEN, Mileto tra Greci e Normanni, in Chiesa e società nel Mezzogiorno. Studi in onore di Maria Mariotti, curr. P. Borzomati et alii, Soveria Mannelli, 1999, p. 109-133: 114.

75 P. DALENA, Calabria medievale, op. cit. (n. 62), p. 143-151; E. CUOZZO, I conti normanni di Catanzaro, in Miscellanea di studi storici, II, 1982, p. 109-127. ${ }^{76}$ P. DALENA, Calabria medievale, op. cit. (n. 62), p. 163-169; D. GIRGENSOHN, Dall'episcopato greco all'episcopato latino nell'Italia meridionale, in La Chiesa greca in Italia dall'VIII al XVI secolo, Atti del convegno storico interecclesiale (Bari, 30 aprile-4 maggio 1969), I, Padova, 1983 (Italia sacra, 20), p. 26-43. Si veda ad esempio il caso di Reggio nel 1086 (KEHR, X, p. 16 e 22; D. STIERNON, Basile de Reggio, le dernier metropolite grec de Calabre, in Rivista di storia della Chiesa in Italia, XVIII (1964), p. 189-226; Cassano nel 1089 (KERH, X, p. 25); di Tropea nel 1094 (KERH, X, p. 37; il diploma è edito da V. CAPIALBI, Sactae Tropaeensis Ecclesiae diplomata expensa, mendis purgata, notisque illustrata, Neapoli, 1840, p. 12-14); di Nicastro nel 1094 (KEHR, X, p. 30) e di Squillace nel 1096 (KEHR, X, p. 55).
} 
nasteri benedettini: Santa Maria della Matina nei pressi di San Marco Argentano 77 , Santa Maria di Sant'Eufemia nella piana lametina ${ }^{78}$ ad opera del Guiscardo, mentre Ruggero patrocinò le fondazioni di Santa Maria dei XII Apostoli nel territorio di Bagnara79, della Certosa di Brunone di Colonia sulle Serre ${ }^{80}$ e della SS. Trinità di Mileto ${ }^{81}$, assestando un colpo pressoché definitivo alle istituzioni religiose bizantine, anche nella Calabria centro meridionale.

L'introduzione del nuovo modello monastico rifletteva il nuovo corso politico voluto dai normanni i quali, non solo portarono abati architetti da Saint Évroult sur Ouche, come nel caso di Roberto di Grandsmenil (o Grantmesnil) ${ }^{82}$ cui si deve il progetto e la realizzazione degli importanti sodalizi monastici, ma anche nuovi modelli di organizzazione del territorio con la creazione di nuovi distretti produttivi attorno alle strutture religiose, dotate di opifici, per riorganizzare la struttura economica della regione ${ }^{83}$.

In questo orizzonte caratterizzato dall'incontro, e dal tentativo di integrazione, di esperienze latine e italo-greche si innestò, nell'XI secolo, l'espansione monastica benedettina; ma furono i normanni, dopo il concilio di Melfi (1059), a promuovere (1062-1091) la fondazione di importanti istituzioni monastiche latine per favorire il recupero dell'ordito ecclesiastico regionale all'obbedienza romana, divenendo strumento delle politiche pontificie di riorganizzazione ecclesiale del Mezzogiorno.

Del resto la storia religiosa dei normanni, oltre a costituire una delle pagine più affascinanti del Mezzogiorno medievale, mette fine e riordina quel mosaico turbolento formato da tantissimi potentati locali. E se ancora non sono totalmente chiare le dinamiche per cui Roberto il Guiscardo, e il fratello Ruggero, riuscirono a realizzare una struttura politica organica nella regione, forse fu la concessione del titolo ducale a consentire loro di chiudere a proprio vantaggio il processo di unificazione politico e religioso normalizzando i «rapporti clanici» e affermando la sovranità sui possedimenti bizantini conquistati e da conquistare.

In questa rinnovata prospettiva politica le fondazioni monastiche e diocesane si rivelarono strumento efficace per il rafforzamento dei poteri ducali di controllo del territorio e dei più importanti snodi viari calabresi, rispondendo contemporaneamente alle esigenze pontificie di riaffermare la sovranità spirituale nel territorio, riorganizzando e rinnovando il tessuto monastico ed episcopale. E, per meglio assolvere a questo compito, tali fondazioni oltre che essere ubicate in posizione strategica lungo la direttrice Capua-Reggio, furono dotate di cospicui patrimoni fondiari la cui gestione, insieme a quella dei lasciti e delle donazioni ricevute, le trasformò in fondamentali cellule dell'economia agraria della regione.

\footnotetext{
77 A. PRATESI, Carte latine, op. cit. (n. 44), p. 3-5 n. 1; L.-R. MENAGER, Recueil des actes des ducs normands d'Italie (1047-1127), I. Les premiers ducs (10461087), Bari, 1981 (Società di storia patria per la Puglia. Documenti e monografie, XLV), p. 65-68, n. 15. All'atto della fondazione seguì immediatamente un nuovo diploma del Guiscardo e della moglie Sikelgaita con il quale venne concessa, all'erigenda abbazia, la facoltà di costruire un nuovo casale per accogliere i villani del casale Pratum e della chiesa di San Venere, la licenza di ricevere donazioni, le esenzioni da corvée, servizi e dal pagamento dell'herbaticum e dell'escaticum, la libertà di pascolo sulle terre ducali e il diritto di giudicare i fuggitivi, rifuggiati nelle terre abbaziali [A. PRATESI, Carte latine, op. cit. (n. 44), p. 7-13, n. 2; L.-R. MENAGER, Recueil des actes, op. cit., p. 68-72, n. 16].

${ }^{78}$ L.-R. MENAGER, Recueil des actes, op. cit. (n. 77), p. 38-47, n. 11; ID., Les fondations monastiques de Robert Guiscard, duc de Pouille et de Calabre, in Quellen und Forschungen aus italianische archiven und bibliotheken, XXXIX (1959), p. 1-116: 4-19; E. PONTIERI, L'abbazia benedettina di Sant'Eufemia nel tempo normanno-svevo, in ID., Tra i normanni nell'Italia meridionale, Napoli, 196433, p. 285-319.

${ }^{79}$ Documenti latini e greci, op. cit. (n. 74), p. 6o-63, n. 9; E. NUCERA, Balnearia e la sua centralità nelle relazioni calabro-sicule dai Normanni all'età moderna, in P. Dalena e B. Saitta (a cura di), op. cit. (n. 7o), p. 147-163.

${ }^{80}$ Documenti latini e greci, op. cit. (n. 74), p. 83-84, n. 14; 138-139, n. 31; 169-183, n. 41-44; 190-192, n. 47. Si vd. anche Documenti dell'Eremo della Torre e del monastero di Santo Stefano del Bosco (secc. XI-XIII), cur. F. Iantorno, Soveria Mannelli, 2009 (Codice diplomatico della Calabria. Serie prima, IV.1), p. 3-4, n. 1; 12-13, n. 5; 15-19, n. 7-8. Si vd. anche il recente lavoro di A. PETERS-CUSTOT, Bruno en Calabre. Histoire d'une fondation monastique dans l'Italie normande, Rome, 2014 (Collection de l'Ėcole française de Rome, 489), p. 57-103 e P. DALENA, Calabria medievale, op. cit. (n. 62), p. 185-190.

${ }^{8}$ Documenti latini e greci, op. cit. (n. 74), p. 35-39, n. 1; L.-R. MENAGER, L'abbaye bénédectine de la Trinità de Mileto, op. cit. (n. 11), p. 9-94: 41-43, n. 13.

${ }^{82}$ Sulla figura dell'abate-architetto si vd. il saggio di G. OCCHIATO, Robert de Grandmesnil: un abate 'architetto' operante in Calabria nell'XI secolo, in Studi medievali, ser. 3, 28 (1987), p. 6o9-666.

${ }^{8}$ Ad esempio la cosiddetta 'prima lega' formata dalla donazione dei territori vicini all'erigenda certosa nelle Serre. La necessità di creare cospicui accumuli territoriali, per dotare le nuove strutture religiose è stato studiato da Sandro Carocci secondo cui proprio i monasteri diventano aree di colonizzazione agricola e di incastellamento favoriti dopo il 1130 dalla protezione della monarchia normanna. Nella fattispecie calabra si tratterebbe di signorie di secondo livello, cioè sottodimensionate rispetto alla geografia signorile del resto del Mezzogiorno e, soprattutto, i dominati locali calabresi non avrebbero concorso alla funzione d'incastellamento perché detentori soltanto di abitati aperti. A tal proposito il riferimento è ai casali ceduti all'abbazia della Matina o quelli donati a Santo Stefano del Bosco [S. CAROCCI, Signorie di Mezzogiorno. Società rurali, poteri aristocratici e monarchia (XII-XIII secolo), Roma, 2014, p. 97-102].
} 